\title{
Compact Ultra-Wideband Bandpass Filter with Variable Notch Characteristics Based on Transversal Signal-Interaction Concepts
}

\author{
Milad Mirzaee, ${ }^{1}$ Bal S. Virdee, ${ }^{2}$ Sima Noghanian ${ }^{1}$ \\ ${ }^{1}$ Department of Electrical Engineering, University of North Dakota, Grand Forks, ND, USA \\ ${ }^{2}$ Faculty of Life Sciences and Computing, Center for Communications Technology, \\ London Metropolitan University, London, United Kingdom
}

Received 8 July 2013; accepted 22 November 2013

\begin{abstract}
A novel technique is presented to design highly compact microstrip ultrawideband (UWB) bandpass filters that exhibit high selectivity quasi-elliptical response. The design is based on transversal signal-interaction concepts that enable the inclusion of single or dual notch-bands within the filter's passband to eliminate interference from other services that coexist within the UWB spectrum. The filter configuration comprises of two transmission paths which include folded T-shaped stepped impedance resonators (SIRs) that are capacitively coupled with the input/output lines to enable signal transmission. It is shown that by combining the filters of different passband centre frequencies an UWB filter can be realised with either a single- or dual-notch function. The theoretical performance of the filter is corroborated via measurements to confirm that the proposed filter exhibits UWB passband of $123 \%$ for a $3 \mathrm{~dB}$ fractional bandwidth, a flat group-delay with maximum variation of less than $0.3 \mathrm{~ns}$, passband insertion loss less than $0.94 \mathrm{~dB}$, high selectivity, a sharp rejection notch-band with attenuation of $\mathbf{- 2 3} \mathbf{d B}$, and a good overall out-of-band performance. Furthermore, the filter occupies a significantly small area of $94 \mathrm{~mm}^{2}$ compared with its classical counterparts. (c) 2014 Wiley Periodicals, Inc. Int J RF and Microwave CAE 24:549-559, 2014.
\end{abstract}

Keywords: bandpass filter (BPF); variable notch-band; ultra-wideband (UWB) filter; stepped impedance resonator (SIR); transversal signal-interaction concepts

\section{INTRODUCTION}

In today's world of wireless communications systems, design of microwave filters has become critical due to increasing demand for advanced communication systems. Examination of recent research literature reveals that ultra-wideband (UWB) applications have aroused considerable interest of academic researchers and $\mathrm{RF} /$ microwave industry since the Federal Communications Commission (FCC) authorized the frequency band from 3.1 to 10.6 $\mathrm{GHz}$ for commercial use [1]. This is very significant as UWB communications technology offers the potential for very high data rate $(>500 \mathrm{Mbit} / \mathrm{s})$ transmission at low transmit power levels making it suitable for emerging short-range technologies. The low power requirement

Correspondence to: M. Mirzaee; e-mail: milad.mirzaee@ my.und.edu.

DOI: $10.1002 /$ mmce. 20798

Published online 6 January 2014 in Wiley Online Library (wileyonlinelibrary.com). necessitates the filter to exhibit low insertion-loss, high selectivity, and flat group delay to minimize distortion of UWB signal. These specifications make the design of UWB filters a challenging task compared with other kinds of microwave filters.

Bandpass filter (BPF) designs for use in UWB wireless communication systems have been implemented using various topologies and employ a variety of procedures, which can generally be classified in three groups, namely: (i) designs based on multiple mode resonators (MMRs) [2-5]; (ii) designs based on cascading a lowpass/bandstop filter with a highpass filter [6-8]; and (iii) designs based on the transversal signal-interaction concepts [9-11]. The introduction of MMRs has supported a new generation of UWB filter designs in the wireless community. A number of MMRs with various structures have been applied in the past few years to model UWB BPFs that contribute a great deal to the success of these crucial microwave components. In [2] and [3], a stepped-impedance MMR comprising of $\lambda / 2$ low-impedance segment at the structure's

(C) 2014 Wiley Periodicals, Inc. 
center with two identical $\lambda / 4$ high-impedance segments on its sides is used to locate its first three resonant modes within the UWB band. In [4] a nonuniform coplanar waveguide MMR with short-circuited ends is proposed to the design of UWB BPF. Another approach is proposed in [5] to realize an UWB filter by loading three open stubs in shunt in the conventional MMR structure at its center and at two symmetrical locations. The resulting UWB filter constructed using the latter approach exploits the first four resonant modes by locating them inside the 3.1-10.6 $\mathrm{GHz}$ UWB band. The higher resonant modes are pushed to higher frequencies to create a wider upper stopband.

A simple approach has been proposed to realize an UWB BPF by cascading a low-pass/band-stop filter with a high-pass filter [6-8]. The main advantage with filters utilizing this design approach is achieving a wide upper stopband; however, the filter posses a relatively large size. The application of transversal signal-interaction concept in the design of microwave filter circuits has already been investigated [9-11]. In these filter structures, the input signal is split and propagates through two different feed-forward signal paths. The design entails forcing signal energy cancellation to produce transmission zeros (TZs) and hence high selectivity and harmonic suppression. In [10], a novel UWB BPF is proposed by cascading two planar marchand baluns, but the selectivity of the filter is not very good and the filter size is relatively large. To improve the selectivity, the authors in [11] proposed a configuration based on cascading a marchand balun with two different transmission lines.

To minimize the size of the transceiver, there is a demand for UWB filters that incorporate a high-attenuation narrowband notch capability. Several structures for UWB BPF with notch-bands have been previously proposed [12-19]. The main shortcoming of some of the cited design approaches is that they yield only a single notch function. Nowadays, the concern is the design of filters whose individual components are capable of generating multiple notch-bands. Some of the filters in this class that were investigated during the last few years are based on multilayer structures [20,21], an asymmetric coupling strip [22], simplified composite right/left-handed resonator [23], defected ground structure (DGS) [24, 25] and folded open stub-loaded MMR [26]. Design of UWB BPF with notch-band using a combination of wideband bandpass filters (BPFs) was first proposed in [15]. The UWB notchband BPF with wider passband, smaller size, and deeper suppression in stopband has been proposed in [27]. The Authors in [28] have used the approach proposed in [15] to create UWB BPF with dual notch-bands, however, the filter is limited by many drawbacks including poor in-band and out-ofband performance, relatively large electrical size, and impedance mismatching at the input and output ports. In this paper, we have refined the design of the UWB BPF proposed in [27] that enables the inclusion of either a single narrow or dual notch-bands within the filter's passband. The UWB BPF is analyzed and a design procedure provided to realize a filter with significantly smaller size as well as wider passband in comparison with its classical counterparts.

\section{FOLDED T-SHAPED STEPPED IMPEDANCE RESONATOR (SIR)}

The typical configuration of a SIR is shown in Figure 1a. The resonator configuration proposed here consists of a folded T-shaped SIR, which can be implemented with either low-impedance or low- and high-impedance segments, as illustrated in Figures $1 \mathrm{~b}$ and 1c, respectively. In the following sections, we demonstrate that the proposed dual-mode resonator contributes towards the realization of compact wideband BPFs when its impedance characteristics and electrical lengths are properly chosen. Compared with other resonators implemented using linear microstrip lines, the proposed resonant structure has the following properties: (i) high degree of compactness; (ii) wide passband capability that is achieved by coupling its resonant modes; (iii) contribution to the design of UWB notch-band bandpass filter; and ability to support a wide stopband by exploiting an appropriate feed structure.

\section{A. Theoretical Modeling of the T-Shaped Resonator Structure}

Initially, we demonstrate the proposed resonator has similar resonance characteristics to a traditional SIR but it is more compact in size. Then we investigate the resonance condition of the proposed resonator by using even-mode and oddmode analysis. A typical SIR shown in Figure 1a consists of a middle low impedance line section with characteristic impedance $Z_{\mathrm{b}}$ and electrical length $\theta_{\mathrm{b}}$ connected to identical high-impedance line sections of characteristic impedance $Z_{\mathrm{a}}$ and electrical length $\theta_{\mathrm{a}}$ on either side of the middle section. When the symmetry plane P-Q in Figure 1a is open-circuit the input admittance of the SIR, which consists of a narrow high-impedance line section of characteristic impedance $Z_{\mathrm{a}}$ and electrical length $\theta_{\mathrm{a}}$ is connected to a wider lowimpedance line section of characteristic impedance $Z_{\mathrm{b}}$ and electrical length $\theta_{\mathrm{b}}$, is given by:

$$
Y_{\text {in }}=1 / Z_{\text {in }}=j Y_{a}\left\{\frac{Y_{a} \tan \theta_{a} \cot \theta_{b}+Y_{b}}{Y_{a} \cot \theta_{b}-Y_{b} \tan \theta_{a}}\right\} .
$$

In this case, the resonator's low-impedance section is split and folded as illustrated in Figure 1b. When the symmetry plane P-Q in Figure 1a is not open-circuit the input admittance of the SIR, is given by:

$$
Y_{\text {in }}=\left\{\frac{j Y_{a} 2\left(Y_{b} \tan \theta_{b}+Y_{a} \tan \theta_{a}\right)\left(Y_{b}-Y_{a} \tan \theta_{a} \tan \theta_{b}\right)}{Y_{a} Y_{b}\left(1-\tan ^{2} \theta_{b}\right)\left(1-\tan ^{2} \theta_{a}\right)-2\left(Y_{a}^{2}+Y_{b}^{2}\right)\left(\tan \theta_{a} \tan \theta_{b}\right)}\right\} .
$$




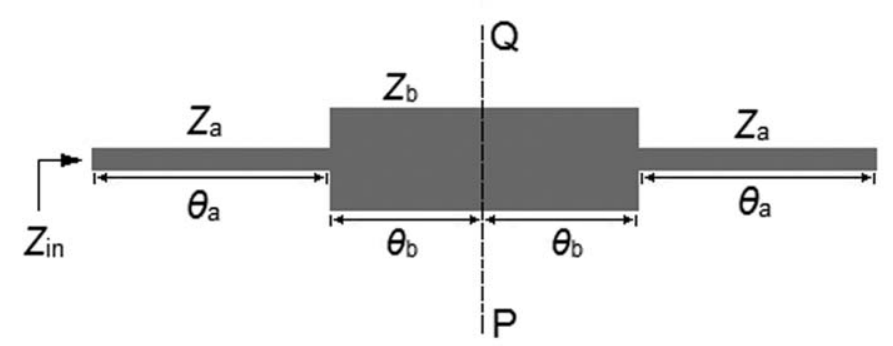

(a)

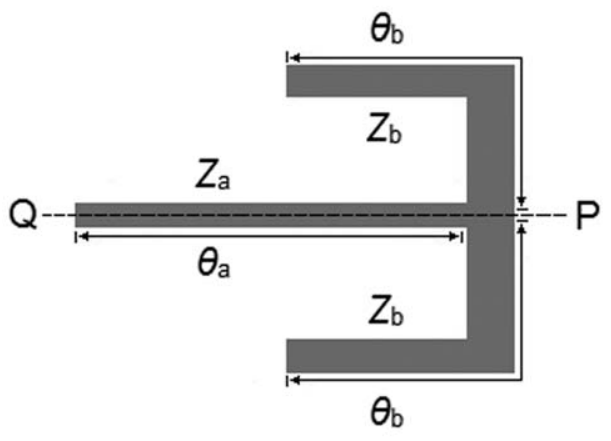

(b)

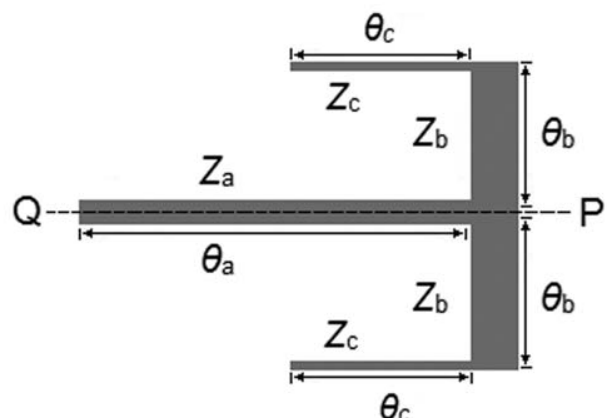

(c)

Figure 1 Three SIR configuration structures; (a) traditional step impedance; (b) proposed folded T-shape with low-impedance segments; and (c) proposed folded T-shape with low-impedance and high-impedance segments.

Under this condition, the basic resonator can be folded as in Figure 1c. Resonance condition is defined when $Y_{\text {in }}$ $=0$. Hence, the resonance mode for the first condition can be determined from the following transcendental equation:

$$
R_{z}=\frac{Y_{b}}{Y_{a}}=-\tan \theta_{a} \cot \theta_{b}
$$

where $R_{z}$ is the impedance ratio of the SIR structure. The ratio of fundamental and higher order resonant frequencies can be determined from (2) in terms of $R_{\mathrm{z}}$ and length ratio $U$ defined as:

$$
U=\frac{\theta_{a}}{\theta_{a}+\theta_{b}}
$$

By substituting (4) into (2), several resonant modes can be found that are dependent on $R_{\mathrm{z}}$ and $U$. By adjusting the impedance ratio $R_{\mathrm{z}}$ and length ratio $U$ it is possible to control the resonant conditions. Thus, it is possible to couple different resonant modes together to form a wideband performance. Once these parameters are determined, the low-impedance segment can be split in half and folded for compactness, as shown in Figure 1b, where the input impedance $Z_{\text {in }}$ is given by:

$$
Z_{i n}=j Z_{a}\left\{\frac{Z_{a} \tan \theta_{a}-\left(\frac{Z_{b}}{2}\right) \cot \theta_{b}}{Z_{a}+\left(\frac{Z_{b}}{2}\right) \cot \theta_{b} \tan \theta_{a}}\right\} .
$$

The two sections of length $\theta_{\mathbf{b}}$ are effectively in parallel. The corresponding input admittance $Y_{\text {in }}$ is given by:

$$
Y_{i n}=j Y_{a}\left\{\frac{2 Y_{b}+Y_{a} \cot \theta_{\mathrm{b}} \tan \theta_{\mathrm{a}}}{Y_{a} \cot \theta_{\mathrm{b}}-2 Y_{b} \tan \theta_{\mathrm{a}}}\right\}
$$

The resonance condition can then be determined using the following transcendental equation:

$$
R_{z}=-\frac{1}{2} \tan \theta_{a} \cot \theta_{b}
$$

This equation indicates the resonator structure in Figure $1 \mathrm{~b}$ has identical resonance characteristics to a typical SIR structure in Figure 1a (when P-Q is open-circuit) with the advantage of using half the impedance ratio to realize a significantly compact design.

\section{B. Even/Odd-Mode Analysis of the Proposed Resonator Structures}

Since the layout of the proposed resonator is symmetrical in the plane P-Q, as indicated in Figure 1, their resonance condition can be analyzed through even-mode and oddmode analysis. In the analysis, the P-Q plane is replaced by an electrical/magnetic wall. For odd-mode excitation, the plane is equal to electrical wall. Figure 2a indicates the approximate equivalent circuit of the proposed resonator in Figure 1b for odd-mode excitation. The input admittance $Y_{\text {inodd }}$ can be expressed as:

$$
Y_{\text {inodd }}=-j Y_{b} \cot \theta_{b} \text {. }
$$

For even-mode excitation, the plane is equal to magnetic wall and the approximate equivalent circuit is 


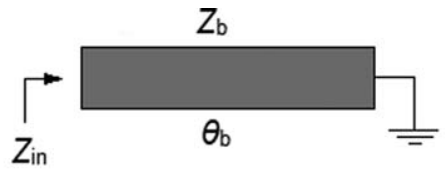

(a)

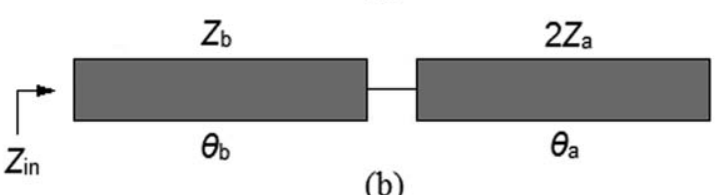

(b)

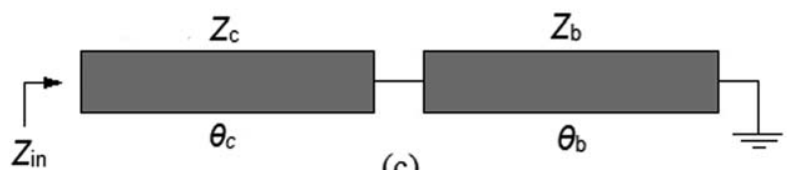

(c)

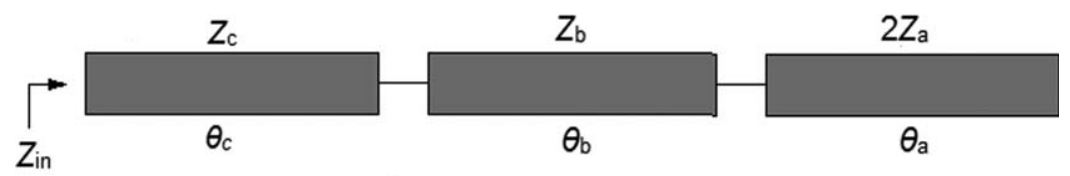

(d)

Figure 2 Equivalent circuit of the proposed resonator configuration in Figure 1b under conditions of: (a) odd-mode and (b) even-mode. Equivalent circuit of Figure 1c under conditions of: (c) odd-mode and (d) even-mode.

illustrated in Figure 2b. The input admittance $Y_{\text {ineven }}$ is given by:

$$
Y_{\text {ineven }}=j \frac{Y_{b}}{2}\left\{\frac{4 Y_{b} \tan \theta_{b}+2 Y_{a} \tan \theta_{a}}{2 Y_{b}-Y_{a} \tan \theta_{a} \tan \theta_{b}}\right\} .
$$

From the condition $Y_{\text {in }}=0$ the fundamental resonances occur at:

For odd mode : $\cot \theta_{b}=0$.

For even mode : $4 Y_{b} \tan \theta_{b}+2 Y_{a} \tan \theta_{a}=0$.

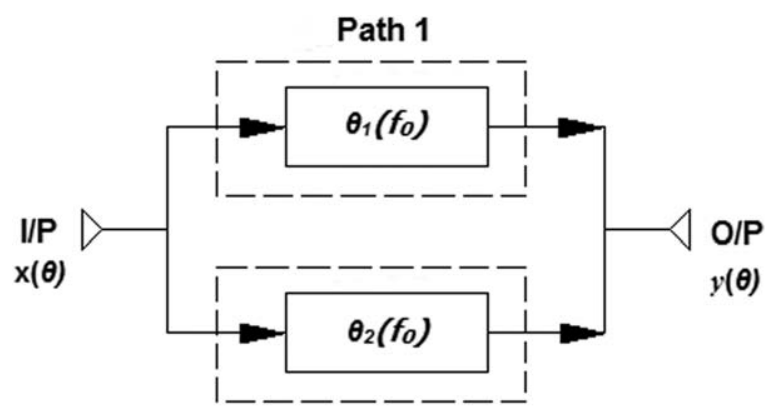

Path 2

Figure 3 Equivalent circuit model of simplified signal interaction concept.
Similarly for the proposed resonator in Figure 1c, the approximate equivalent circuit are shown in Figures $2 \mathrm{c}$ and $2 \mathrm{~d}$ for even-mode and odd-mode, respectively. The input admittance $Y_{\text {inodd }}$ is calculated as follow:

$$
Y_{\text {inodd }}=j Y_{c}\left\{\frac{Y_{c} \tan \theta_{b} \tan \theta_{c}-Y_{b}}{Y_{c} \tan \theta_{b}+Y_{b} \tan \theta_{c}}\right\} .
$$

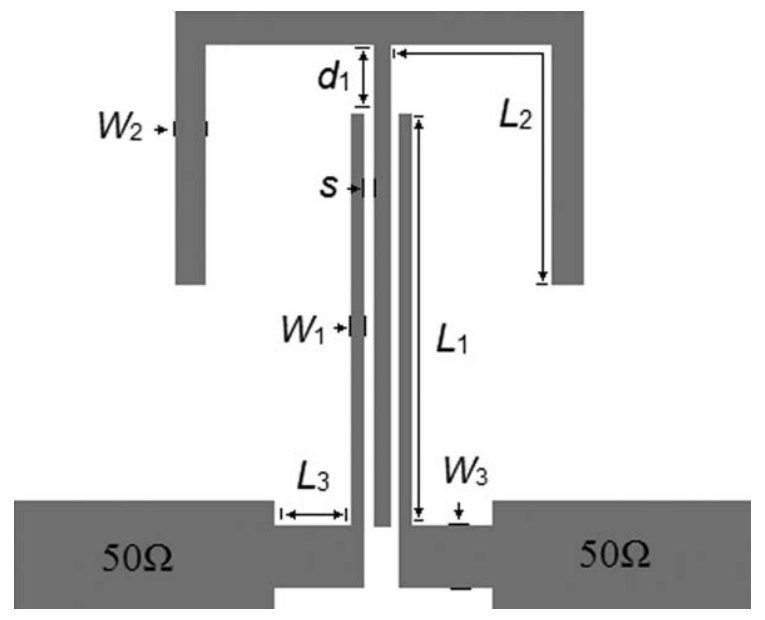

Figure 4 Layout of prototype wideband filter. The optimized parameters are: $L_{1}=11.1, d_{1}=3.2, L_{2}=3.7, L_{3}=0.5, W_{1}=0.1$, $W_{2}=0.5$, and $\mathrm{W}_{3}=1$ (dimensions are in millimeters). 


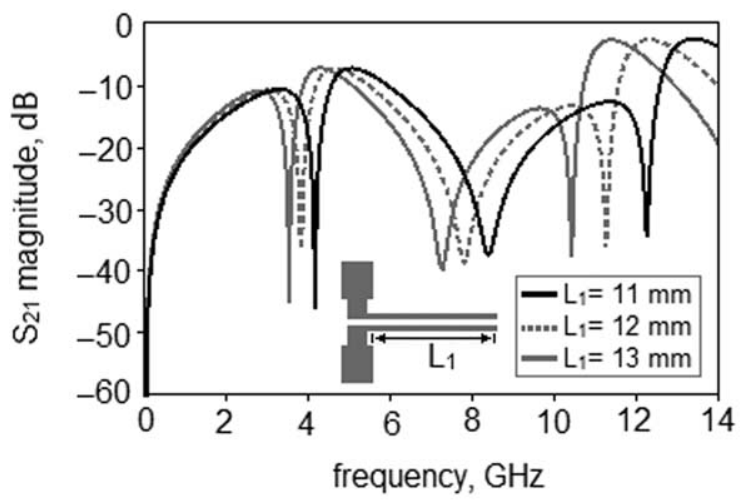

(a)

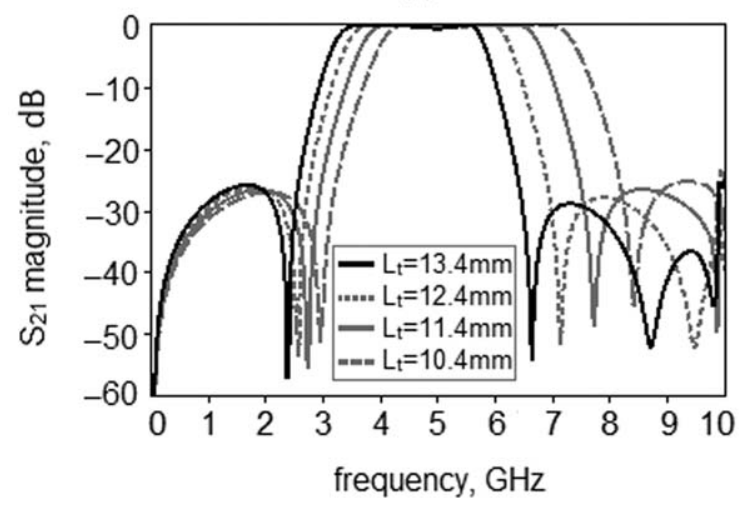

(c)

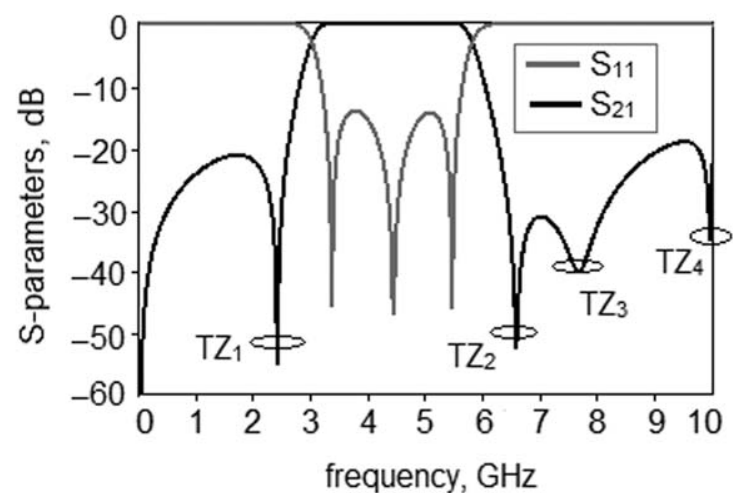

(b)

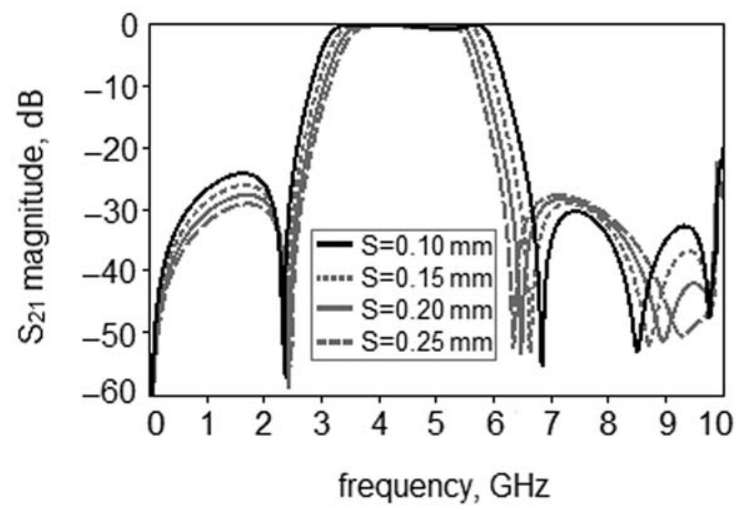

(d)

Figure 5 Simulated insertion-loss response of the proposed wideband BPF: (a) $S_{21}$ of the feed structure as a function of $L_{1}$; (b) S-parameters of the proposed wideband BPF; (c) location of TZs, which depend on length $L_{\mathrm{t}}$ (where $L_{\mathrm{t}}=L_{1}+d_{1}$ ); and (d) the affect on the FBW as a function of coupling gap $(s)$.

And the input admittance $Y_{\text {ineven }}$ can be given by:

$$
Y_{\text {ineven }}=j Y_{c}\left\{\frac{Y_{b}\left(Y_{a} \tan \theta_{a}+2 Y_{b} \tan \theta_{b}\right)+Y_{c} \tan \theta_{c}\left(2 Y_{b}-Y_{a} \tan \theta_{a} \tan \theta_{b}\right)}{Y_{c}\left(2 Y_{b}-Y_{a} \tan \theta_{a} \tan \theta_{b}\right)-Y_{b} \tan \theta_{c}\left(Y_{a} \tan \theta_{a}+2 Y_{b} \tan \theta_{b}\right)}\right\} .
$$

Then resonance conditions are given by:

For odd mode:

$$
Y_{c} \tan \theta_{b} \tan \theta_{c}-Y_{b}=0 .
$$

For even mode:

$Y_{b}\left(Y_{a} \tan \theta_{a}+2 Y_{b} \tan \theta_{b}\right)+Y_{c} \tan \theta_{c}\left(2 Y_{b}-Y_{a} \tan \theta_{a} \tan \theta_{b}\right)=0$.

From the above equations, it can be concluded that resonance conditions can be controlled by adjusting electrical lengths of the resonator's structure for both even and oddmodes. These specifications can be utilised for the design of wideband bandpass filters with adjustable passbands.

\section{SIMPLIFIED TRANSVERSAL SIGNAL-INTERACTION CONCEPT}

Figure 3 shows the equivalent circuit model of simplified signal interaction concept where the input signal is split and propagates through two feed-forward signal paths with different electrical lengths. The signal transmission can be given by [29]:

$$
\begin{aligned}
& y(\theta)=\left[h_{1}\left(\theta_{1}\right)+h_{2}\left(\theta_{2}\right)\right] \cdot x(\theta) \\
& h_{1}\left(\theta_{1}\right)=e^{i \theta_{1}}, \quad h_{2}\left(\theta_{2}\right)=e^{i \theta_{2}}
\end{aligned}
$$

Based on the transversal filter concepts, a passband and stopband can be achieved by the following relations [29]:

$$
\begin{array}{ll}
\theta_{1}\left(f_{0}\right)=\theta_{2}\left(f_{0}\right) \pm 2 n \pi, & (n=0,1,2 \ldots) \\
\theta_{1}\left(f_{0}\right)=\theta_{2}\left(f_{0}\right) \pm n \pi, & (n=1,3,5 \ldots)
\end{array}
$$

where $f_{0}$ is the center frequency of the filter. As will be shown in the next section the proposed filter configuration 


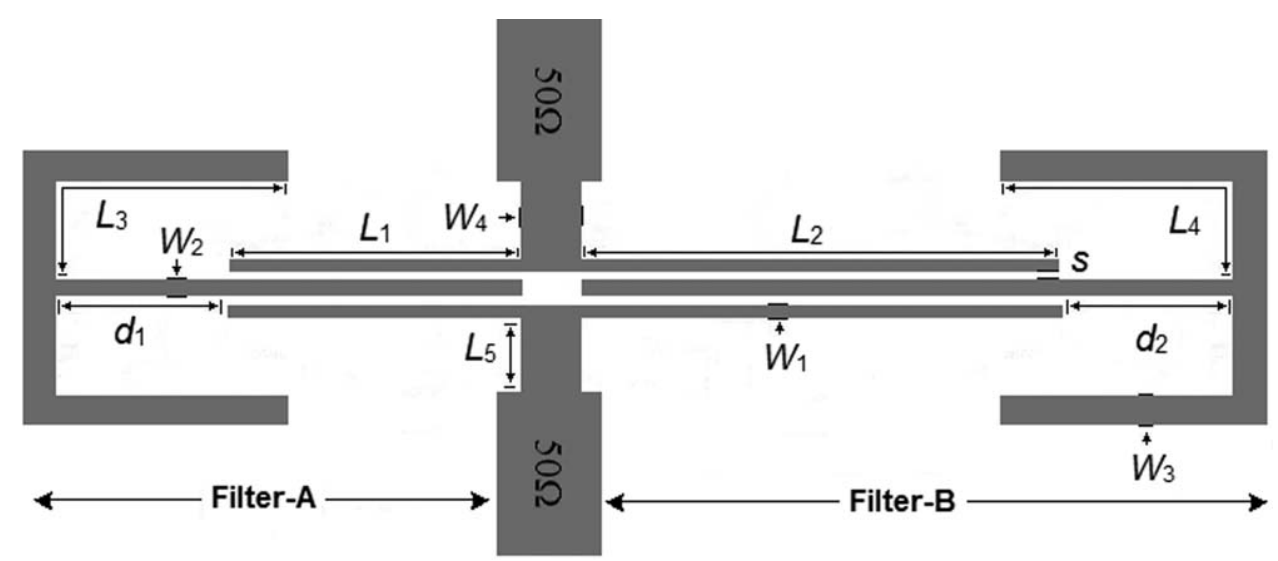

(a)

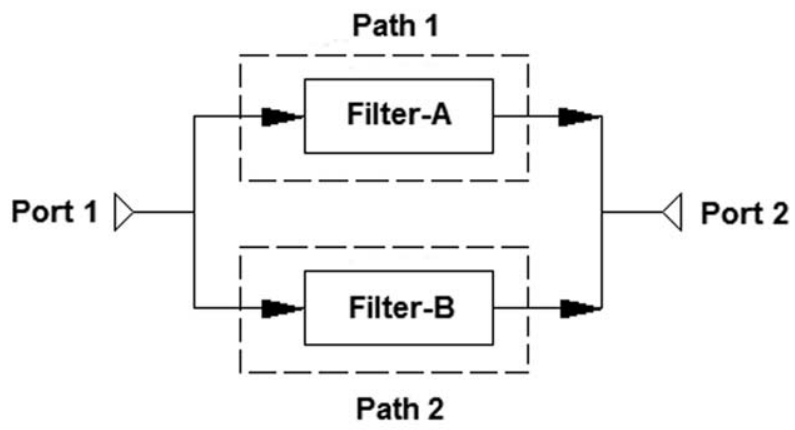

(b)

Figure 6 (a) Layout of one UWB BPF with single-notch function and (b) signal flow through the filter structure.

is located in the two paths and are coupled with the input/ output ports. The parameters of the filter structure and its coupling with the input/output ports are exploited to control its bandwidth and generate notch-bands.

\section{PROTOTYPE WIDEBAND BPF DESIGN}

For the proposed resonator in Figure $1 \mathrm{~b}$ it is evident from (10) that the odd resonant modes depend on the length $\theta_{\mathrm{b}}$ of the low-impedance line with corresponding length of $L_{2}$, which is given by [30]:

$$
f_{\text {odd }}=\frac{c}{4 L_{2} \sqrt{\varepsilon_{\text {eff }}}}
$$

where $c$ is the speed of light in the free space, and $\varepsilon_{\text {eff }}$ denotes the effective dielectric constant of the microstrip line. The relationship shows the odd-mode resonant frequencies are inversely dependent on length proportional to length $\theta_{\mathrm{b}}$. The resonant frequencies of even-mode excitation depend on the length of $\theta_{\mathrm{a}}$ with corresponding length of $L_{\mathrm{t}}$ (where $L_{\mathrm{t}}=L_{1}+d$ ). Similarly as $\theta_{\mathrm{a}}$ increases the even-mode resonant frequencies move towards lower frequencies. The dimensions of the T-shape structure gener- ates resonant modes and the first two modes are coupled together in the configuration proposed in Figure 4 to create a wide passband. The input/output feed structure creates TZs, which are a function of the length of coupling arms. With this structure it is possible to suppress higher resonant modes and achieve a wide stopband. Figure 5a indicates the insertion-loss response of the input/output coupling structure, which is a function of $L_{1}$. It shows the transmission response resulting from the input/output structure without the T-shaped resonator. It should be noted that a parallelcoupling feed structure with a cross coupling can contribute to strong coupling and therefore low passband insertionloss as well as additional TZs in the region of the spectrum above and below the passband skirts [27].

As it is evident from Figure 5a, this cross-coupled feed structure is capable of producing multiple TZs, which are distributed among the lower and upper stopband region. The location of TZs can easily be controlled by varying the length of $L_{1}$. It can be clearly observed that by decreasing $L_{1}$, while other optimized parameters remain fixed, the frequency of the TZs move towards upwards in frequency. In addition, the proposed structure has intrinsic transmission zero whose location can be controlled by varying $L_{\mathrm{t}}$. Figure 5 b shows an excellent $S_{21}$ response of the wideband filter structure. It has four TZs distributed around 2.39, 6.57, 


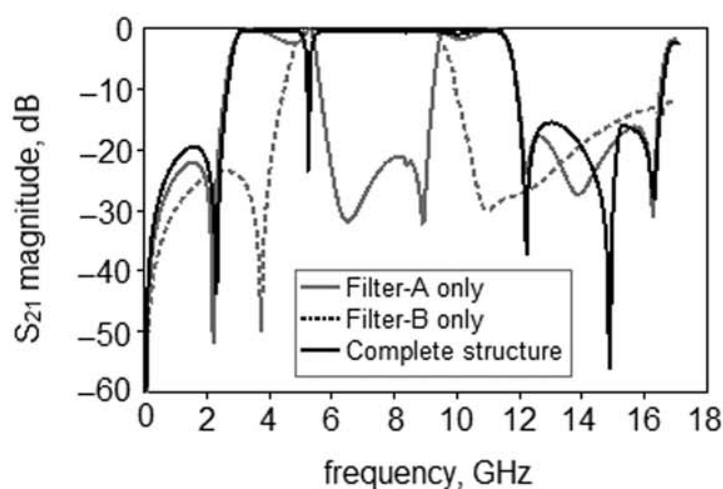

(a)

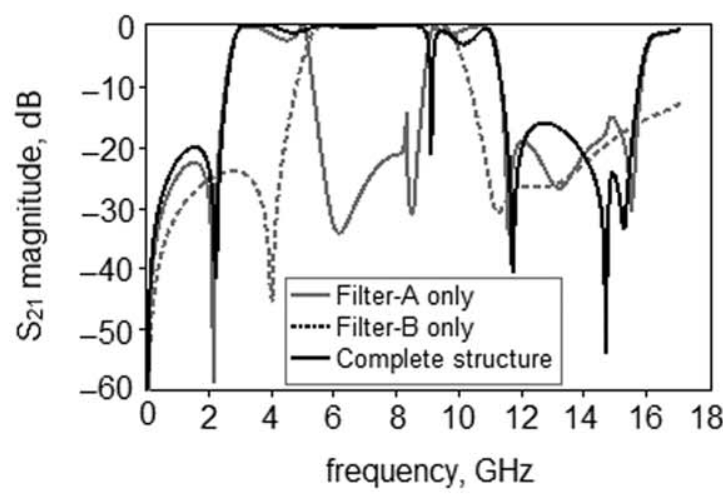

(c)

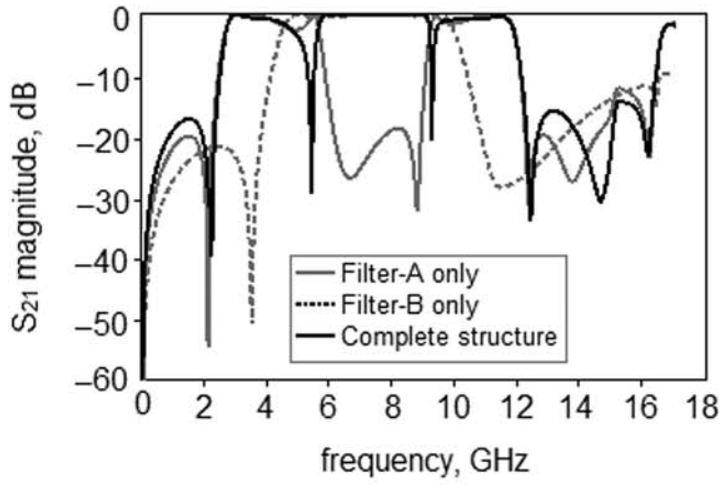

(e)

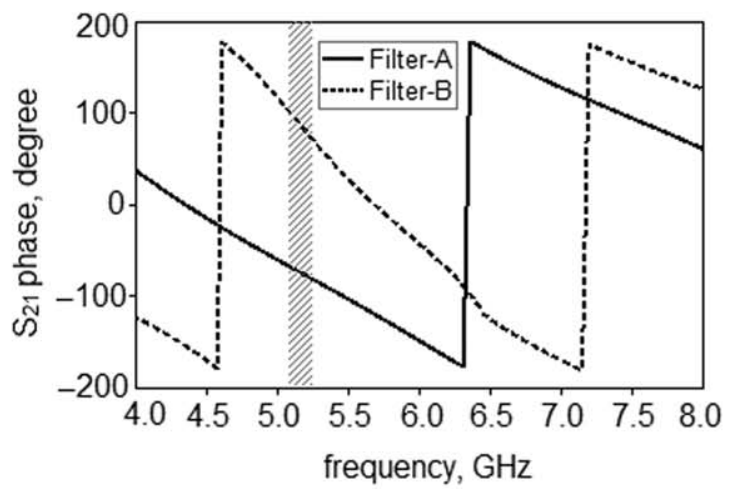

(b)

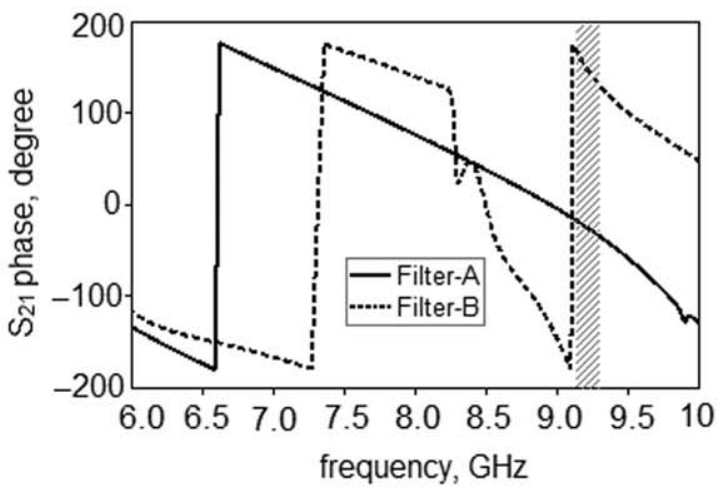

(d)

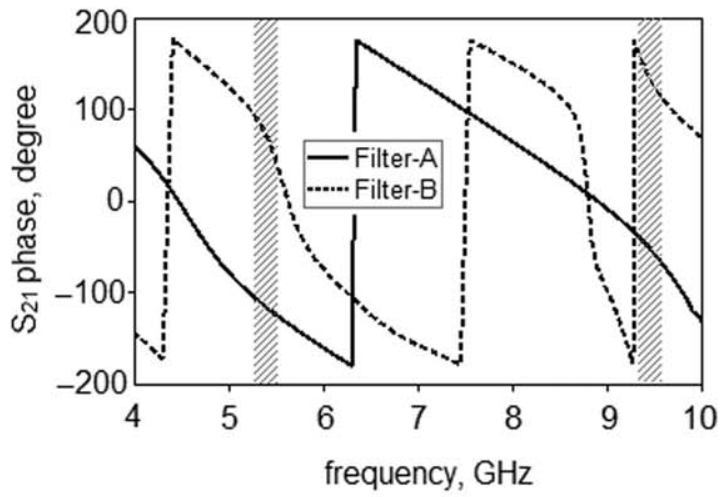

(f)

Figure 7 a and b Show the simulated insertion-loss and phase response, respectively, of the UWB filter in Figure 6a composed of Filter$\mathrm{A}$ and Filter-B. The structure creates a notch-band at the lower end of the passband response; (c) and (d) show $S_{21}$ and phase response, respectively, of the UWB filter with a notch-band at the upper end of the passband; and (e) and (f) show $S_{21}$ and phase response of UWB filter with dual notch-bands. (Note: shaded areas indicate the regions of suppression).

7.65, and $9.95 \mathrm{GHz}$, which contribute a great deal to the high selectivity and good upper stopband performance. In addition, three transmission poles within the passband are almost balanced, which has the potential to provide balanced wideband passband behavior. As was discussed earlier, by changing $\theta_{a}$ with corresponding length of $L_{\mathrm{t}}$, the even resonant modes decrease in frequency while the odd modes remain fixed. It is evident from Figure $5 \mathrm{c}$ by changing $L_{\mathrm{t}}$ it is feasible to change the location of TZs and consequently the bandwidth of the passband. By increasing $L_{\mathrm{t}}$ from 10.4 to $13.4 \mathrm{~mm}$, the passband is shifted toward lower frequencies. In addition, the filter's fractional bandwidth (FBW) can be controlled by adjusting the coupling gap $(s)$ between the resonator and the capacitive cross-coupled feed structure. This is evident in Figure $5 \mathrm{~d}$, by merely increasing $s$ from 0.1 to $0.25 \mathrm{~mm}$, the FBW decreases from 64 to $45 \%$. 


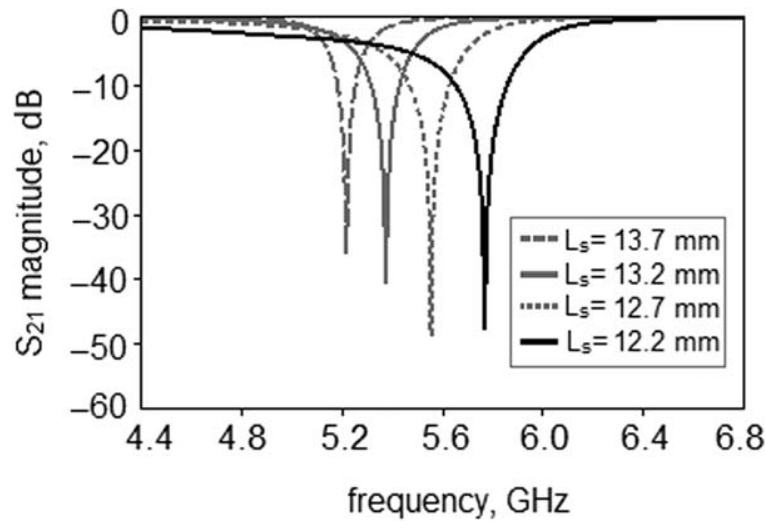

Figure 8 Center frequency of notch-band as a function of length $L_{\mathrm{s}}\left(L_{\mathrm{s}}=L_{2}+d_{2}\right)$.

\section{UWB BPF WITH SINGLE/ DOUBLE NOTCH-BANDS}

Figure $6 \mathrm{a}$ shows the layout of the proposed UWB filter with its physical parameters. Figure $6 \mathrm{~b}$ depicts the schematic diagram of the proposed filter where the input signal propagate from port 1 to port 2 along paths 1 and 2 through the wideband filters Filter-A and Filter-B developed using the proposed design in Section IV. To achieve an UWB BPF with excellent performance and including a sharp notch-band, the filters with a different passband center frequency were connected as depicted in Figure 6a. The mechanism of creating the UWB passband with narrow notch-bands was implemented using the transversal signal interaction concepts. A narrow rejection band is implemented by forcing the signal propagating on paths 1 and 2 to cancel at the output port, which is achieved when the signal magnitudes are equal with a phase differential of $180^{\circ}$. This structure is capable of producing single narrow notch-band or dual notch-bands at low and high frequencies simultaneously. The frequency and phase response of the UWB filter in Figure 6a with a notchband at the lower end of the filter's response are shown in Figures $7 \mathrm{a}$ and $7 \mathrm{~b}$, respectively [27]. As can be seen from Figure $7 \mathrm{a}$, when the $S_{21}$ response of Filter-A overlaps with that of Filter-B, this creates a region of suppression resulting in a narrow and sharp notch-band at around 5.21 GHz. Moreover, it is evident from Figure 7b, the insertion-loss of Filter-A and Filter-B have a phase difference about $180^{\circ}$ in the suppression region as predicted by the transversal signal-interaction concept.

To generate the notch at a higher frequency, the $S_{21}$ response of Filter-A was shifted upwards by decreasing the length of $L_{\mathrm{t}}$ so that it overlapped with the response of Filter-B. Thus, a narrow notch-band was created at a higher frequency, as shown in Figure 7c. As is evident in Figure $7 \mathrm{~d}$, the phase difference between Filter-A and Filter-B is about $180^{\circ}$ in the suppression region as well. According to the discussion in previous section, by decreasing the coupling gap $(s)$ between the folded $\mathrm{T}$ shaped resonator and the input/output lines the bandwidth of the prototype wideband filter can be increased. Hence, by decreasing the coupling gap in the proposed UWB structure, the $S_{21}$ response of Filter-A overlaps with that

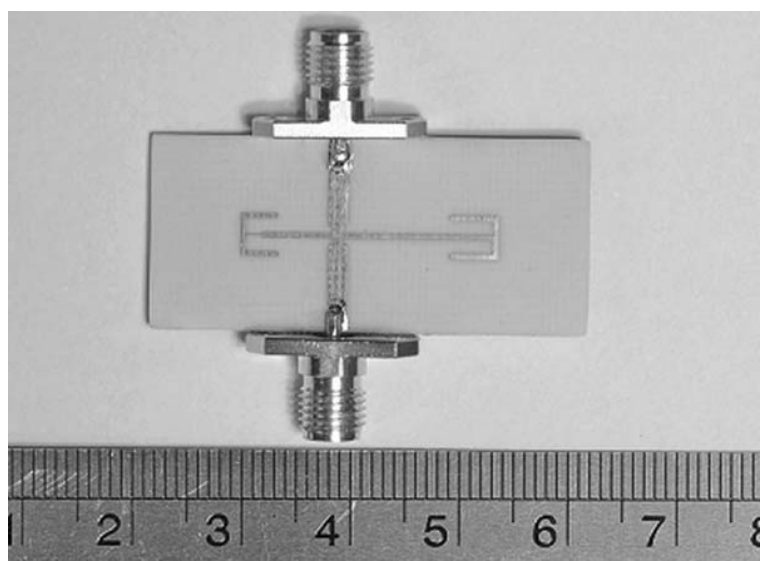

Figure 9 Photograph of the fabricated filter.

of Filter-B at a lower and upper frequency to simultaneously create two narrow notch-bands within the UWB passband, as shown in Figure 7e. The structure was optimized using Agilent's advance design system (ADS). The optimized parameters to achieve a high-performance UWB BPF with a single notch are: $L_{1}=6.5, d_{1}=1.4$, $L_{2}=13.4, d_{2}=0.3, L_{3}=4.4, L_{4}=5.4, L_{5}=0.5, W_{1}=0.1$, $W_{2}=0.2, W_{3}=0.5, W_{4}=1$, and $s=0.1$ (all dimensions

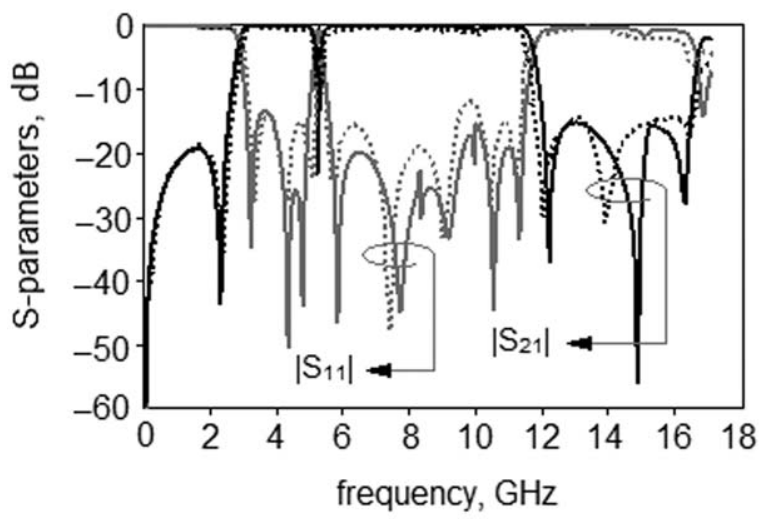

(a)

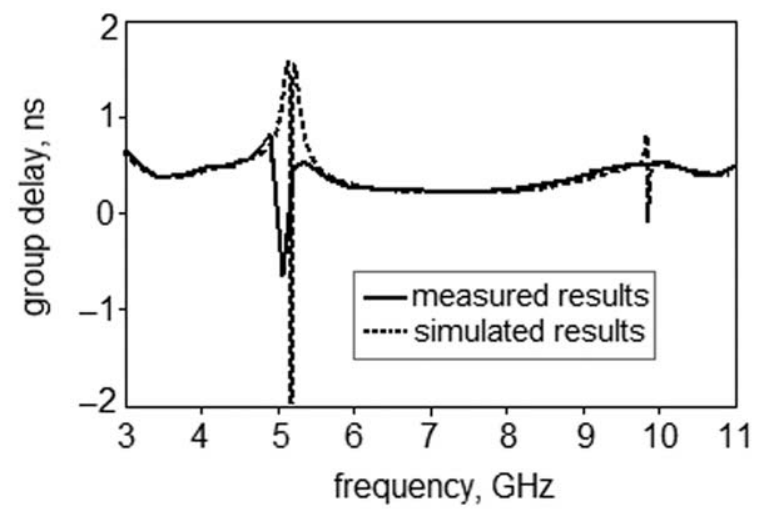

(b)

Figure 10 (a) Measured and simulated S-parameter results of the proposed filter and (b) group-delay. 
TABLE I Comparison of the Proposed Filter with Best Reported UWB BPFs

\begin{tabular}{|c|c|c|c|c|c|c|}
\hline Ref. & Area $\left(\mathrm{mm}^{2}\right)$ & $\mathrm{FBW}(\%)$ & $\begin{array}{l}\text { Insertion/ } \\
\text { return-loss (dB) }\end{array}$ & Group-Delay (ns) & $\begin{array}{l}\text { Single-/dual-notch } \\
\text { capability }\end{array}$ & Structure \\
\hline [2] & 16.9 & $113 \%$ & $0.55 / 10.00$ & 0.23 & no/no & MMR \\
\hline [3] & 29.9 & $110 \%$ & $1.30 / 14.00$ & 0.30 & no/no & MMR \\
\hline [5] & 37.12 & $114 \%$ & $1.40 / 14.30$ & 0.64 & no/no & MMR \\
\hline [10] & 840 & $110 \%$ & $1.35 / 10.50$ & 0.50 & no/no & Marchand balun \\
\hline [11] & 450 & $110 \%$ & $1.20 / 12.00$ & 0.50 & no/no & Marchand balun + TL \\
\hline$[12]$ & 180.8 & $110 \%$ & $0.90 / 11.05$ & 0.27 & yes/no & SIR stubs \\
\hline [13] & 170.8 & $103.2 \%$ & $0.45 / 12.00$ & 0.60 & yes/yes & Parasitic coupled line \\
\hline [14] & 576 & $117.6 \%$ & $1.00 / 10.00$ & 0.20 & yes/no & DGS \\
\hline [15] & 127.9 & $101.9 \%$ & $0.37 / 14.18$ & Not given & yes/no & Folded SIR \\
\hline [16] & 160.4 & $107.7 \%$ & $0.80 / 12.00$ & Not given & yes/no & Radial stub \\
\hline [17] & 191.8 & $114.5 \%$ & $0.83 / 10.08$ & 0.29 & yes/no & MMR + slotline \\
\hline [18] & 78.8 & $114.5 \%$ & $0.50 / 10.00$ & 0.50 & yes/no & CRLH \\
\hline [19] & 59.5 & $124 \%$ & $1.10 / 10.00$ & 0.19 & yes/no & Iultiple slotline resonaors \\
\hline [20] & 300.8 & $120.4 \%$ & $0.75 / 15.00$ & 0.40 & no/yes & Multilayer LCP \\
\hline [21] & 405.7 & $106.3 \%$ & $0.66 / 10.00$ & 0.20 & yes/yes & Multilayer LCP \\
\hline [22] & 63.7 & $118.8 \%$ & $0.80 / 12.50$ & 0.20 & no/yes & symmetric coupling strip \\
\hline [23] & 680 & $118.2 \%$ & $>1.0 / 10.00$ & Not given & no/yes & SCRLH \\
\hline [24] & 60.3 & $110 \%$ & $0.46 / 15.00$ & 0.32 & yes/yes & Surface-coupled \\
\hline [25] & 220 & $108.5 \%$ & $2.00 / 15.00$ & Not given & no/yes & TL + defected SIR \\
\hline [26] & 88 & $114 \%$ & $1.00 / 12.00$ & Not given & yes/yes & MMR \\
\hline This work & 94 & $123.2 \%$ & $0.94 / 12.00$ & 0.30 & yes/yes & Folded SIR \\
\hline
\end{tabular}

given are in millimeters) [27]. The input/output line of 50 $\Omega$ lines are matched to the resonant structure via high impedance line of length $L_{5}$ and width $W_{4}$. By adjusting $L_{1}+d_{1}, L_{2}+d_{2}$, and the coupling gap $(s)$, the notch response can be adjusted relatively easily. As indicated in Figure 8 , by decreasing $L_{\mathrm{s}}\left(L_{\mathrm{s}}=L_{2}+d_{2}\right)$ from 13.7 to $12.2 \mathrm{~mm}$, while keeping all other parameters fixed, the notch frequency can be made to shift towards the higher frequency. The rejection level of the notch can effectively mitigate interference signals from $5 \mathrm{GHz}$ WLAN IEEE 802.11a systems.

\section{SIMULATED AND MEASURED RESULTS}

Full-wave electromagnetic simulation tool (ADS) has been used to analyze the design of the proposed filter. Moreover, the final prototype was fabricated on the substrate with thickness of $32 \mathrm{mil}$ and dielectric constant of 3.38 to experimentally verify the theoretical results of the proposed design.

By use of the optimized physical dimensions, a super performance compact UWB BPF with notch-band was fabricated and its performance measured. The S-parameters were measured using an Agilent 8722ES network analyzer. The photograph of the fabricated filter is shown in Figure 9.

Figure 10 shows the measured and simulated S-parameter response and group-delay of filter [27]. These results confirm the proposed filter exhibits a wide passband from 2.66 to $11.2 \mathrm{GHz}$, with a corresponding FBW of $123 \%$ for $S_{11}>10 \mathrm{~dB}$, which covers FCC mask. The response is accompanied by a narrow bandstop notch with a FBW of $8.8 \%$ from 4.98 to $5.44 \mathrm{GHz}$, and peak rejection of $-23.38 \mathrm{~dB}$. In addition, the flat group-delay is achieved except at the notch-band with maximum variation of $<0.3$ ns. The discrepancy between the measured and simulated group-delay is attributed to the fabrication tolerance and inaccuracy of the CAD models. The stopband has width of $4.75 \mathrm{GHz}$, with a relative stopband bandwidth of $34 \%$. The proposed filter exhibits a quasi-elliptical response with maximally flat passband response. The simulated and measured insertion-loss is $<0.27$ and $0.94 \mathrm{~dB}$, respectively, and the return-loss is better than $12 \mathrm{~dB}$ across most of the passband. The actual area of the filter is $94 \mathrm{~mm}^{2}$. Table I gives a comparison of the proposed design in terms of size and performance with a number of best previously reported UWB filters. The FBW and stopband are enhanced by around 20 and $23 \%$, respectively, and size is reduced around $26 \%$ in comparison with design presented in [15]. The proposed filter satisfies the stringent requirements of next generation of UWB telecommunication systems.

\section{CONCLUSION}

By exploiting proposed folded T-shaped SIR resonator, a very compact dual-mode wideband BPF has been realised. An UWB filter was created by combining two of these wideband filters. The resulting UWB filter possessed significantly enhanced out-of-band rejection compared with currently published UWB filters. It was shown notchbands can be introduced within the filter's passband response enabling the rejection of interfering signals at 5 $\mathrm{GHz}$ from WLAN IEEE 802.11a systems. The mechanism of creating notch-bands was delineated using the transversal signal-interaction concept. The filter possesses a very wide passband of $123 \%$ for a $3 \mathrm{~dB}$ factional bandwidth, a flat group-delay, relatively low insertion-loss $(<0.94 \mathrm{~dB})$, high selectivity, sharply rejection notch-band $(-23 \mathrm{~dB})$, and a good overall out-of-band performance. These 
features make the proposed filter structure a commercially viable for numerous broadband wireless communications and radar systems.

\section{REFERENCES}

1. Federal Communications Commission (FCC), Revision of part 15 of the commission's rules regarding ultra-wideband transmission systems, First Report and Order, FCC 02-48, 2002.

2. L. Zhu, S. Sun, and W. Menzel, Ultra-wideband bandpass filters using multiple-mode resonator, IEEE Microwave Wireless Comp Lett 15 (2005), 796-798.

3. S. Sun and L. Zhu, Capacitive-ended interdigital coupled lines for UWB bandpass filters with improved out of band performances, IEEE Microwave Wireless Comp Lett 16 (2006), 440-442.

4. J. Gao, L. Zhu, W. Menzel, and F. Bogelsack, Short circuited CPW multiple-mode resonator for UWB bandpass filter, IEEE Microwave Wireless Comp Lett 16 (2006), 104-106.

5. R. Li and L. Zhu, Compact UWB bandpass filter using stubloaded multiple-mode resonator, IEEE Microwave Wireless Comp Lett 17 (2007), 40-42.

6. W. Menzel, M.S. Rahman Tito, and L. Zhu, Low-loss ultrawideband (UWB) filters using suspended stripline, Proc AsiaPacific Microwave Conf 4 (2005), 2148-2151.

7. R. Gomez-Garcia, and J.I. Alonso, Systematic method for the exact synthesis of ultra-wideband filtering responses using highpass and low-pass sections, IEEE Trans Microwave Theory Tech 54 (2006), 3751-3764.

8. C-W. Tang, and M-G. Chen, A microstrip ultra-wideband bandpass filter with cascaded broadband bandpass and bandstop filters, IEEE Trans Microwave Theory Tech 55 (2007), 2412-2418.

9. C. Rauscher, Microwave active filters based on transversal and recursive principles, IEEE Trans Microwave Theory Tech 33 (1985), 1350-1360.

10. W.J. Feng, and W.Q. Che, Ultra-wideband bandpass filter using broadband planar marchand balun, Electron Lett 47 (2011), 198-199.

11. W.J. Feng, W.Q. Che, and T.F. Eibert, Ultra-wideband bandpass filter based on transversal signal-interaction concepts, Electron Lett 47 (2011), 1330-1331.

12. P. Mondal, and Y.L. Guan, A coplanar stripline ultrawideband bandpass filter with notch band, IEEE Microwave Wireless Comp Lett 20 (2010), 22-24.

13. S. Pirani, J. Nourinia, and C. Ghobadi, Band-notched UWB BPF design using parasitic coupled line, IEEE Microwave Wireless Comp Lett 20 (2010), 444-446.

14. G-M. Yang, R. Jin, C. Vittoria, V.G. Harris, and N.X. Sun, Small ultra-wideband (UWB) bandpass filter with notched band, IEEE Microwave Wireless Comp Lett 18 (2008), 176178.

15. M. Mirzaee, A novel small ultra-wideband bandpass filter including narrow notched band utilizing folded T-shaped stepped impedance resonator, Prog Electromagn Res C 22 (2011), 85-96.

16. J. Xu, W. Wu, W. Kang, and C. Miao, Compact UWB bandpass filter with a notched band using radial stub loaded resonator, IEEE Microwave Wireless Comp Lett 22 (2012), 351353.

17. C.-H. Lee, C.-I.G. Hsu, and C.-J. Chen, Band-notched balanced UWB BPF with stepped-impedance slotline multi-mode resonator, IEEE Microwave Wireless Comp Lett 22 (2012), $182-184$.

18. K.U. Ahmed and B.S. Virdee, Ultra-wideband bandpass filter based on composite right/left handed transmission-line unitcell, IEEE Trans Microwave Theory Tech 61 (2013), 782788 .

19. K. Song, T. Pan, and Q. Xue, Compact ultra-widebnad notchband bandpass filters using multiple slotline resonators, Microwave Opt Tech Lett 54 (2012), 1132-1135.

20. Z.-C. Hao and J.-S. Hong, Compact UWB filter with double notch-bands using multilayer LCP technology, IEEE Microwave Wireless Comp Lett 19 (2009), 500-502.

21. Z.-C. Hao, J.-S. Hong, J.P. Parry, and D.P. Hand, Ultra-wideband bandpass filter with multiple notch bands using nonuniform periodical slotted ground structure, IEEE Trans Microwave Theory Tech 57 (2009), 3080-3088.

22. K. Song and Q. Xue, Compact ultra-wideband (UWB) bandpass filters with multiple notched bands, IEEE Microwave Wireless Comp Lett 20 (2010), 447-449.

23. F. Wei, Q.Y. Wu, X.W. Shi, and L. Chen, Compact UWB bandpass filter with dual notched bands based on SCRLH resonator, IEEE Microwave Wireless Comp Lett 21 (2011), 2830.

24. J.-Q. Huang, Q.-X. Chu, and C.-Y. Liu, Compact UWB filter based on surface-coupled structure with dual nothched bands, Prog Electromagn Res 106 (2010), 311-319.

25. P. Sarkar, R. Ghatak, M. Pal, and D.R. Poddar, Compact UWB bandpass filter with dual notch bands using open circuited stubs, IEEE Microwave Wireless Comp Lett 22 (2012), 453-455.

26. M. Mirzaee, A novel compact UWB BPF with independently controllable double notched bands and super wide upper stopband, J. Electromagnet Wave 27 (2012), 77-86.

27. M. Mirzaee and B.S. Virdee, UWB bandpass filter with notch-band based on transversal signal-interaction concepts, IET Electron Lett 49 (2013), 399-401.

28. M. Nosrati and M. Daneshmand, Compact microstrip UWB double/single notch-band BPF based on wave's cancellation theory, IET Microwave Antennas Propag 6 (2012), 862868.

29. W. Che, Applications of transversal signal-interaction concept in ultra-wideband filter circuits, IEEE International High Speed Intelligent Communication Forum (HSIC) 2012. Invited paper.

30. K. Song, and Q. Xue, Novel broadband bandpass filters using Y-shaped dual-mode microstrip resonators, IEEE Microwave Wireless Comp Lett 19 (2009), 448-550. 


\section{BIOGRAPHIES}

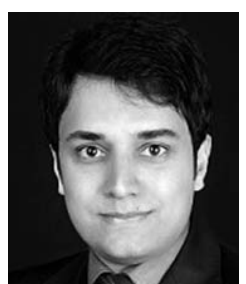

Milad Mirzaee earned his B.Sc. degree in electrical engineering in 2008. He is currently working toward the M.Sc. degree at the Applied Electromagnetics Engineering Lab, Department of Electrical Engineering, University of North Dakota, USA.

His B.Sc. thesis, on the design and fabrication of a painter robot, achieved first place in technical challenge in the AmirKabir University of Technology National Robotic Competition, Tehran, Iran, in 2009 and qualified for the Second International Amirkabir University of Technology Robotic Competitions and the Third National Khwarizmi Robotic Competitions in 2010.

Since 2008, he has worked on the design and fabrication of microwave passive components. He has published several technical articles and reports in prestigious scientific journals and magazines, and has made presentations on his research work at leading international technical conferences. His research covers a wide range of areas, including antennas and RF, microwave, and millimeter-wave circuits and systems for next-generation wireless communications applications. He is a reviewer of IEEE Microwave and Wireless Component Letters.

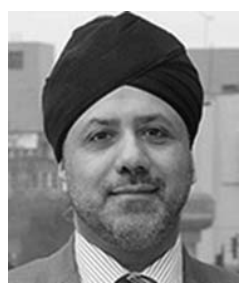

Bal S. Virdee received the B.Sc. (Hons) and MPhil degrees in Communications Engineering from the University of Leeds, UK and his Ph.D. in Electronic Engineering from the University of London, UK. He has worked in industry for various companies including Philips (UK) as an R\&D engineer and Filtronic Components Ltd. as a future products developer in the area of $\mathrm{RF} / \mathrm{micro}-$ wave communications. He has taught at several academic institutions before joining London Metropolitan University where he is a Professor of Microwave Communications in the Faculty of Life Sciences and Computing where he Heads the Center for Communications Technology and is the Director of London Metropolitan Microwaves. His research, in collaboration with industry and academia, is in the area of microwave wireless communications encompassing mobile phones to satellite technology. Prof.Virdee has chaired technical sessions at IEEE international conferences and published numerous research papers. He is Executive Member of IET's Technical and Professional Network Committee on RF and Microwave Technology. He is a Fellow of IET and a Senior Member of IEEE.

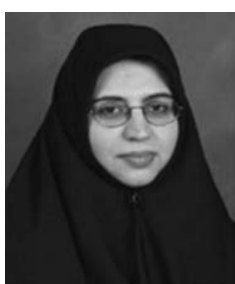

Sima Noghanian received the B.Sc. degree in electrical engineering from the Sharif University of Technology, Tehran, Iran, in 1992, and the M.Sc. and Ph.D. degrees, both in electrical engineering, from the University of Manitoba, Winnipeg, Canada, in 1996 and 2001, respectively. In 2001, she was a researcher at YottaYotta Corporation, Edmonton, Canada. She received a Postdoctoral Fellowship from Natural Sciences and Engineering Research Council of Canada in 2002, which she took at the University of Waterloo. She was an Assistant Professor in the Department of Electrical Engineering, Sharif University of Technology, Iran, during 2002-2003. From 2003 to 2008, she was an Assistant Professor in the Department of Electrical and Computer Engineering, University of Manitoba, Canada. She is currently an Associate Professor in the Department of Electrical Engineering, University of North Dakota, USA. Her research interests include antenna design and modelling, wireless channel modelling, ultra-wideband antennas, and microwave imaging. Dr. Noghanian is a senior member of IEEE and served as the IEEE Winnipeg Waves Chapter (joint Chapter of Antenna \& Propagation, Microwave Theory \& Techniques, and Vehicular Technology societies) Chair during 20042005. She is a member of International Union of Radio Science URSI Commission B, and a member of the Applied Computational Electromagnetics Society. She is currently an area editor for AEUInternational Journal of Electronics and Communications. 